\title{
Seismic Attenuation and Velocity Dispersion to Discriminate Gas Hydrates and Free Gas Zone, Makran Offshore, Pakistan
}

\author{
Muhammad Irfan Ehsan"1*, Perveiz Khalid², Nisar Ahmed², Jiachun You', Xuewei Liu1, \\ Tahir Azeem ${ }^{1}$ \\ ${ }^{1}$ School of Geophysics and Information Technology, China University of Geosciences (Beijing), Beijing, China \\ ${ }^{2}$ Institute of Geology, University of the Punjab, Lahore, Pakistan \\ Email: *irfan_opq@yahoo.com
}

Received 16 July 2016; accepted 16 August 2016; published 19 August 2016

Copyright (C) 2016 by authors and Scientific Research Publishing Inc.

This work is licensed under the Creative Commons Attribution International License (CC BY). http://creativecommons.org/licenses/by/4.0/ (c) (i) Open Access

\begin{abstract}
Gas hydrates gained a remarkable attention as an unconventional energy resource recently. In order to interpret gas hydrates (part of fluid) and free gas saturated zone accurately, it is essential to implement new technique related to seismic attenuation and velocity dispersion. $P$ wave attenuation and velocity dispersion in porous media made promising imprints for exploration of gas hydrates. The most prominent phenomenon for attenuation and velocity dispersion in porous media is wave induced fluid flow in which wave inhomogeneities are larger than pore size but smaller than wavelength. Numerical simulation technique is applied to analyze frequency dependent velocity dispersion and attenuation in gas hydrates and free gas layer in Makran offshore of Pakistan. Homogeneous and patchy distribution patterns of gas hydrates and free gas within pore spaces of host sediments at lower and higher frequency regime are considered. It is noted that the attenuation and velocity dispersion increase with the increase in gas hydrates saturation. The maximum attenuation is observed at $66 \%$ saturation of gas hydrates in the area under investigation. However, in case of water and gas mixture the maximum attenuation and velocity dispersion occur at low gas saturation $(\sim 15 \%)$. Therefore, based on our numerical simulation, velocity dispersion and attenuation can be used as seismic attributes to differentiate various gas saturations and gas hydrates saturation for Makran offshore area of Pakistan.
\end{abstract}

\section{Keywords}

Gas Hydrates, Seismic Attenuation, Velocity Dispersion, Makran Offshore, Seismic Attributes

\footnotetext{
${ }^{*}$ Corresponding author.
}

How to cite this paper: Ehsan, M.I., Khalid, P., Ahmed, N., You, J.C., Liu, X.W. and Azeem, T. (2016) Seismic Attenuation and Velocity Dispersion to Discriminate Gas Hydrates and Free Gas Zone, Makran Offshore, Pakistan. International Journal of Geosciences, 7, 1020-1028. http://dx.doi.org/10.4236/ijg.2016.78077 


\section{Introduction}

Gas hydrate is a potential future energy resource in which huge quantity of methane gas is trapped as guest molecule in water molecule [1] [2]. These unconventional energy resources are found at continental margin and permafrost [3]. Gas hydrates have different distribution and occurrence patterns, for example they may exist as a part of host sediments and part of fluids [4]. Usually gas hydrates are inferred on seismic section by a strong reflector known as bottom simulating reflector (BSR) [5]-[8].

Since last decade, seismic attenuation and velocity dispersion gained a reasonable attention to explore gas hydrates reservoir [9] [10]. The seismic attenuation in hydrate-bearing sediments depends largely on the distribution pattern of hydrates. Pecher and Holbrook [11] described that when gas hydrates are part of solid, they will cause an increase in seismic attenuation, but Rosi [12] negated their suggestions and recommended that when gas hydrates are part of solid, they may decrease seismic attenuation. Interpretation of sonic wave form data from Mackenzie (Canada) shows that seismic attenuation increases with the presence of gas hydrates-bearing sediments [13]. Vertical seismic profile data from Malikwell (Canada) show a strong attenuation response for gas hydrate saturated sediments [14]. In contrary, Matsushima [15] and Sain [16] concluded that when gas hydrates are part of solid they will incredibly stiffer host sediments, which is the major cause of decrease in attenuation with gas hydrate concentration. Li [17] endorsed the attenuation results of Sain [16] by doing numerical simulation for gas hydrates bearing sediments in Shenhu area of China. All above-mentioned researchers underestimate the attenuation phenomena for gas hydrates-bearing sediments when gas hydrates are considered part of formation fluid.

The Makran offshore of Pakistan has a large potential of gas hydrates [18] [19]. Ehsan [18] studied the seismic and elastic properties as a function of gas hydrates saturation and distribution patterns. They studied these properties without considering seismic attenuation and velocity dispersion. In the present study, seismic wave attenuation and velocity dispersion phenomena are used to discriminate gas hydrates and free gas zones from water saturated sediments in Makran offshore of Pakistan. Location map of study area has shown in (Figure 1). The behavior of $P$ wave velocity for gas layer at low frequency is nearly a straight line after certain saturation limit [18]. It is essential to find out the technique which will differentiate accurately reservoir saturation zone at low frequency domain. So we tried to implement seismic attenuation technique to sort out gas layer and gas hydrates saturation zone. A fruitful effort is made by Khalid and Ahmed [20] to understand the phenomenon of velocity dispersion and attenuation in partially saturated reservoirs. The velocity dispersion and attenuation in gas hydrates-bearing sediments of Makran offshore are studied under wave induced fluid flow (WIFF) mechanism at mesoscopic scale [20] and effective medium theory [21].

\section{Work Flow and Methodology}

In order to elaborate variation in elastic properties such as saturated bulk modulus of gas hydrates and gasbearing sediments in uniform and patchy distribution patterns, we have used two approaches: Gassmann-Wood and Gassmann-Hill in two frequency domains i.e. low and high respectively [20] [22]. At low frequency patch,

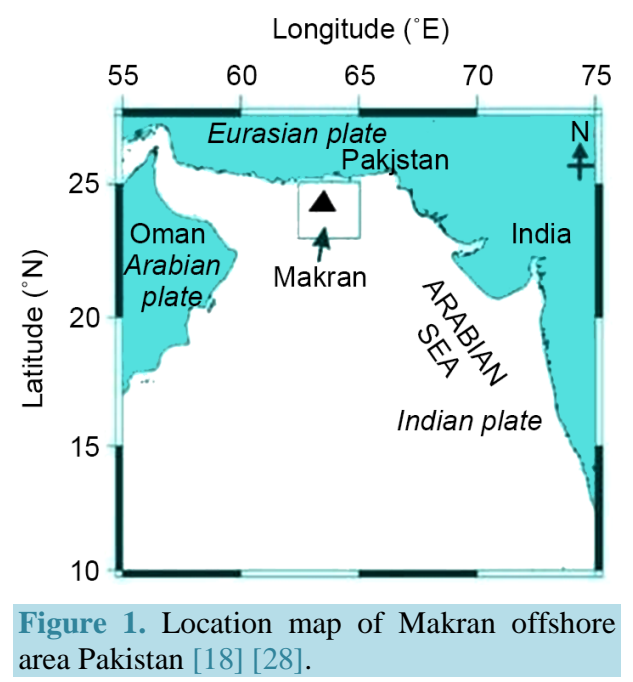


size is smaller than wave length and seismic wave has enough time for pore pressure equilibration. We have used Wood's average [23] or Reuss's average approach [24] to compute bulk modulus of gas hydrate/water and gas/water layer at low frequency regime [20].

$$
K_{f_{0}}=\left[\frac{X_{w}}{K_{w}}+\frac{X_{h}}{K_{h}}\right]^{-1} .
$$

In the above equation, $X_{w}$ and $X_{h}$ are the saturation of water and hydrate. In order to compute response of bulk modulus for gas layer at low frequency, we simply alter $X_{h}$ by $X_{g}$. We have used effective medium theory and modified form of Gassmann's equation [25] to compute saturated bulk modulus for gas hydrates/water and gas/water layer at low frequency regime

$$
K_{\text {sat }_{o}}=K_{m} \frac{\phi K_{d r y}-(1+\phi) K_{f_{o}} K_{d r y} / K_{m}+K_{f_{o}}}{(1-\phi) K_{f_{o}}+\phi K_{m}-K_{f_{o}} K_{d r y} / K_{m}}
$$

In the above equation, $K_{f_{o}}$ is the accumulative bulk modulus of fluid at low frequency and $K_{d r y}$ is the bulk modulus of dry sediments, $K_{m}$ is accumulative bulk modulus of minerals and $\phi$ is the porosity of rock matrix. $P$ wave modulus at low frequency limit $\left(P_{\text {sat } 0}\right)$ for gas hydrates and gas-bearing sediments is given below.

$$
P_{\text {sat } 0}=K_{\text {sat } 0}+4 \mu / 3 \text {. }
$$

In Equation (7) $\mu$ is shear modulus of dry sediments. In our modeling we have considered gas hydrates as a part of fluid so shear modulus of dry sediments equal to shear modulus of saturated sediments.

$P$ wave velocity $\left(\alpha_{0}\right)$ at low frequency regime (relaxed state) is given as

$$
\alpha_{0}=\left(P_{\text {sat } 0} / \rho_{b}\right)^{1 / 2} .
$$

In order to compute velocity dispersion (variation of seismic wave velocity in porous medium with respect to frequency) and attenuation for gas hydrates and gas bearing sediments in Makran offshore area of Pakistan. The input parameters derived from Equations (1)-(4), the attenuation and velocity dispersions can be modeled by using equations described below [26].

$$
\begin{gathered}
V_{\text {phase }}(f)=\sqrt{\operatorname{Re}\left[P_{\text {sat }}(f) / \rho_{b}\right]} . \\
1 / Q(f)=\operatorname{Im}\left[P_{\text {sat }}(f)\right] / \operatorname{Re}\left[P_{\text {sat }}(f)\right] .
\end{gathered}
$$

Further the relative variation in $P_{\text {sat }}$ and $\alpha$ which can be computed as [20].

$$
\begin{gathered}
\Delta P_{\text {sat }} / P_{\text {satoo }}=\left(P_{\text {sato }}-P_{\text {sat } 0}\right) / P_{\text {sato }} . \\
\Delta \alpha / \alpha_{\infty}=\left(\alpha_{\infty}-\alpha_{0}\right) / \alpha_{\infty} .
\end{gathered}
$$

When higher frequencies seismic wave propagates through gas hydrates-bearing sediments or gas saturated layer, the wave length of seismic wave is smaller than patch size. Thus, the stiffness of pore fluid increased and cause an increase in velocity dispersion. We have used Ehsan [18] and Hill [27] methodology to compute seismic response of gas hydrates-bearing sediments.

\section{Results}

Our main goal of this study is to understand the effect of elastic properties on velocity dispersion and attenuation for gas hydrates-bearing sediments, when they occurred as a part of fluid. The input parameters used in this numerical modeling are given in Table 1 . The $P$ wave velocity difference for gas hydrates-bearing sediments computed by GW (low frequency) GH approaches presented in (Figure 2) as a function of gas hydrates saturation. Previous study made by Ehsan [18] shows that the $P$ wave velocity in low frequency regime is lower than of high frequency regime. At extreme points $\left(S_{h} \sim 0\right.$ and $\left.S_{h} \sim 1.0\right)$ where pores are fully saturated with single fluid, the velocity dispersion is zero whereas at $0<S_{h}>1.0$ both approaches give different values of velocity. The maximum velocity difference is attained at $S_{h} \sim 0.66$ where velocity difference is up to $50 \mathrm{~m} / \mathrm{s}$. This difference can be seen on high resolution seismic data in the form of velocity dispersion. 
Table 1. Parameters used for numerical simulation to compute seismic attenuation and velocity dispersion (Ehsan et al., 2015 [18]).

\begin{tabular}{cccc}
\hline Parameters & Symbols & Numerical values & Units \\
\hline Porosity & $\Phi$ & 39 & $\%$ \\
Viscosity of water & $\left(\eta_{w}\right)$ & 0.005 & Poise \\
Viscosity of gas & $\left(\eta_{g}\right)$ & 0.00021 & Poise \\
Number grains per contact & $N$ & 9 & \\
Gas hydrate bulk modulus & $K_{h}$ & 6.41 & $\mathrm{GPa}$ \\
Gas hydrate shear modulus & $G_{h}$ & 2.54 & $\mathrm{GPa}$ \\
Gas hydrate density & $\rho_{h}$ & 0.91 & $\mathrm{~g} / \mathrm{Cm}{ }^{3}$ \\
Gas bulk modulus & $K_{g}$ & 0.067 & $\mathrm{GPa}$ \\
Gas density & $\rho_{g}$ & 0.20 & $\mathrm{~g} / \mathrm{cm}^{3}$ \\
Water bulk modulus & $K_{W}$ & 2.25 & $\mathrm{GPa}$ \\
Density of water & $\rho_{w}$ & 1.0 & $\mathrm{~g} / \mathrm{cm}^{3}$ \\
\hline
\end{tabular}

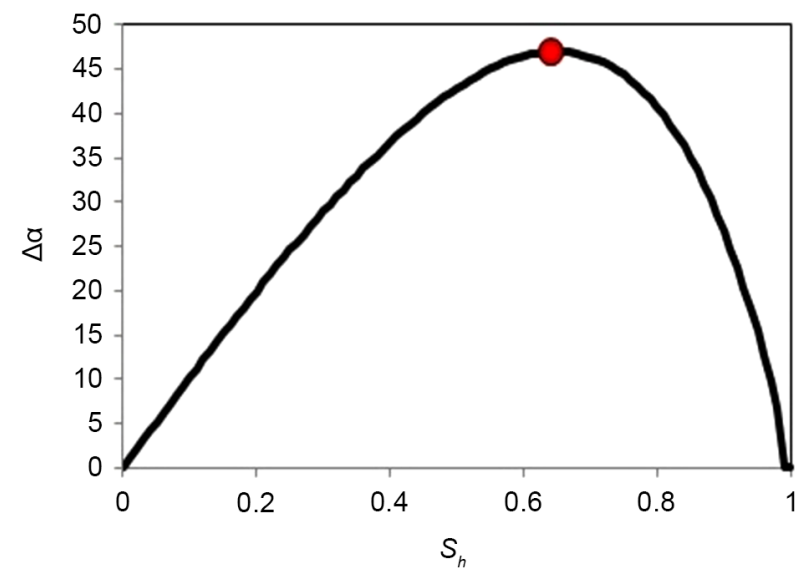

Figure 2. Velocity difference plot against gas hydrates saturation for GW and GH approach.

Similarly, the velocity dispersion, when fluid distribution pattern depart from homogenous to patchy saturation, in partially gas-saturated sediments as a function of gas saturation $\left(S_{g}\right)$ is demonstrated in the (Figure 3). It has been observed that the GW method underrate the seismic velocities. Stiffness of gas bearing sediments is much higher during patchy saturation. Velocity difference trend between lower frequency (Wood) and high frequency (Hill) domains shows totally different pattern from gas hydrate bearing sediments. Velocity difference $(\Delta \alpha)$ curve against gas saturation shows the maximum difference at low gas saturation (almost at 15\%) and starts decreasing gradually as the gas saturation increases (Figure 3). In case of single fluid phase (100\% water or gas saturation), both the approaches give similar results and therefore the velocity difference became zero.

In the (Figure 4(a) and Figure 4(b)), we have plotted the difference in elastic modului of fluids versus gas hydrates and gas saturation for both approaches (GW and GH) respectively. In case of gas hydrates-water case the maximum difference arise again at $S_{h} \sim 66 \%$ ( $\mathrm{Sw} \sim 34 \%$ ), however in case of gas-water fluid phases, the maximum difference appears at low gas saturation (Sg 15\%).

In the Figure 4(a) shows that $\Delta K_{f}$ for gas hydrate increases with increasing gas hydrate saturation. After $66 \%$ gas hydrate saturation $\Delta K_{f}$ starts decreasing. In (Figure 4(b)), we have seen that $\Delta K_{f}$ response for gas layer starts increasing with the increase of gas saturation, after $15 \%$ saturation, it starts decreasing. Now we have checked relative difference response of $P$ wave modulus $\left(\Delta P_{\text {sat }} / P_{\text {sato }}\right)$ and velocity $\left(\Delta \alpha / \alpha_{\infty}\right)$ for gas hydrate and gas 


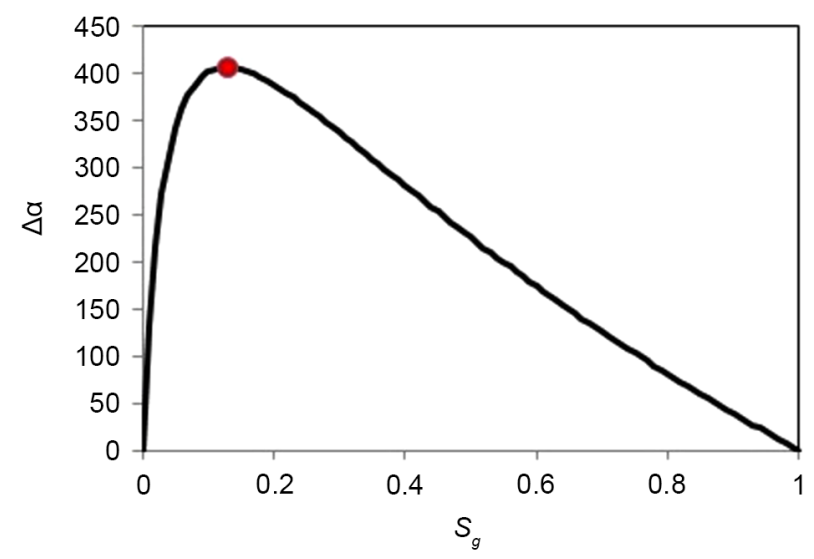

Figure 3. Velocity difference plot for gas saturated zone with GW and GH approach.

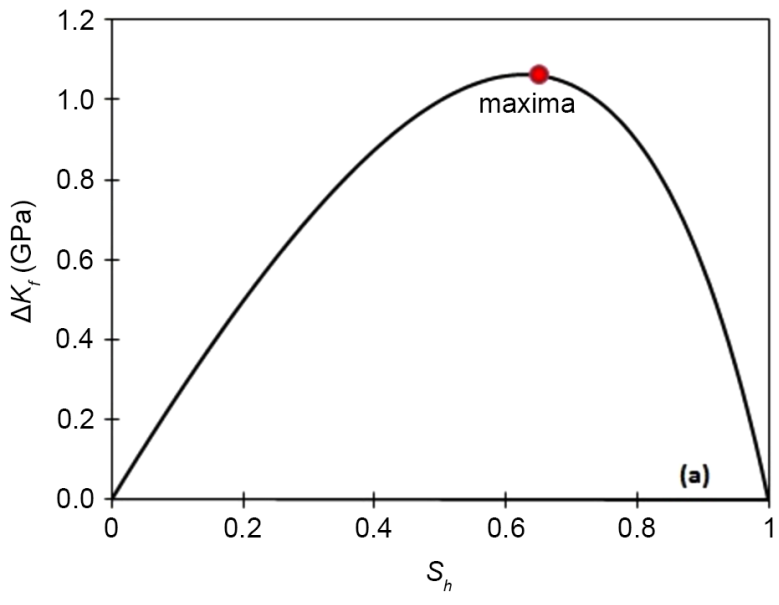

(a)

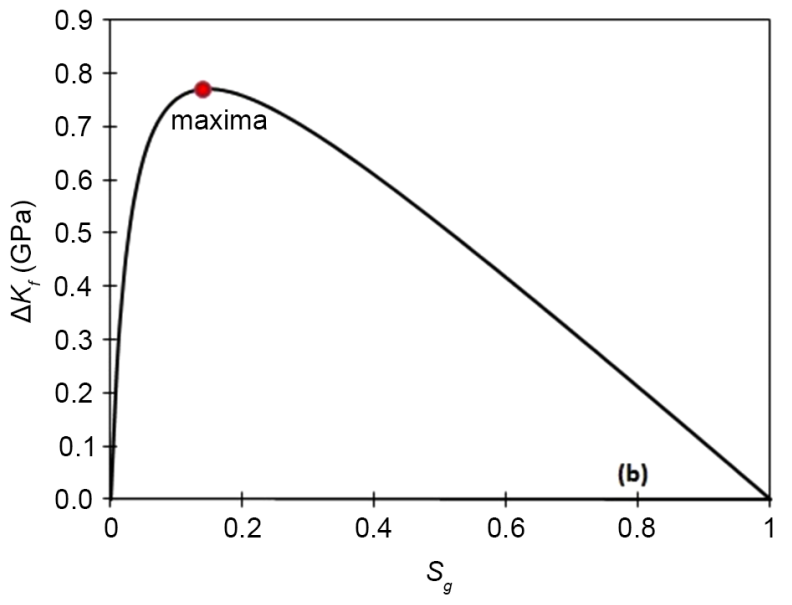

(b)

Figure 4. (a) Bulk modulus of fluids difference response for homogeneos and patchy distribution of gas hydrates bearing sediments (b) Bulk modulus of fluids difference response for homogeneos and patchy distribution of gas saturated sediments.

bearing saturated sediments. It is observed that at $0 \%$ and $100 \%$ of gas and gas hydrate saturation, relative difference of velocity and $P$ wave modulus shows minimum response. It is clear from (Figure 5) that maximum difference of $P$ wave modulus and velocity is obtained at $66 \%$ of gas hydrate saturation and $15 \%$ of gas saturation. It is clear from (Figure 5(a)) $P$ wave modulus relative difference response and $P$ wave velocity relative difference response for gas hydrates saturated sediments initially increase with increasing gas hydrates saturation and it will start decreasing after $66 \%$ gas hydrates saturation. Similar variation response is observed for gas saturated sediments in (Figure 5(b)).

\subsection{Seismic Attenuation and Velocity Dispersion}

In our previous discussion we have mentioned the variation in elastic properties for gas hydrates and gas saturation. Now we are trying to elaborate the imprints of these elastic properties to sort out their effects on seismic attenuation and velocity dispersion for gas hydrates and gas bearing sediments. The velocity dispersion and attenuation plots have been drawn at different saturations of gas hydrates in (Figure 6).

Velocity dispersion plots for different gas hydrates saturation versus water saturations are shown in (Figure 6(a)). In the (Figure 6(a)) lower extremity is representing homogeneous phase and upper extremity representing patchy phase. Here it is essential to mention that in case of patchy saturation, we have considered two patches, which are saturated with a symmetrical scheme, one patch saturated with water and other patch saturated with 


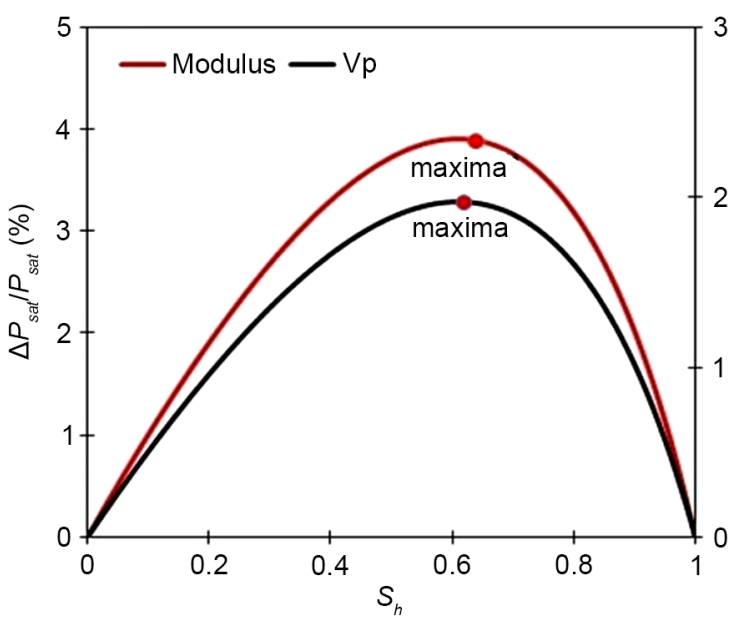

(a)

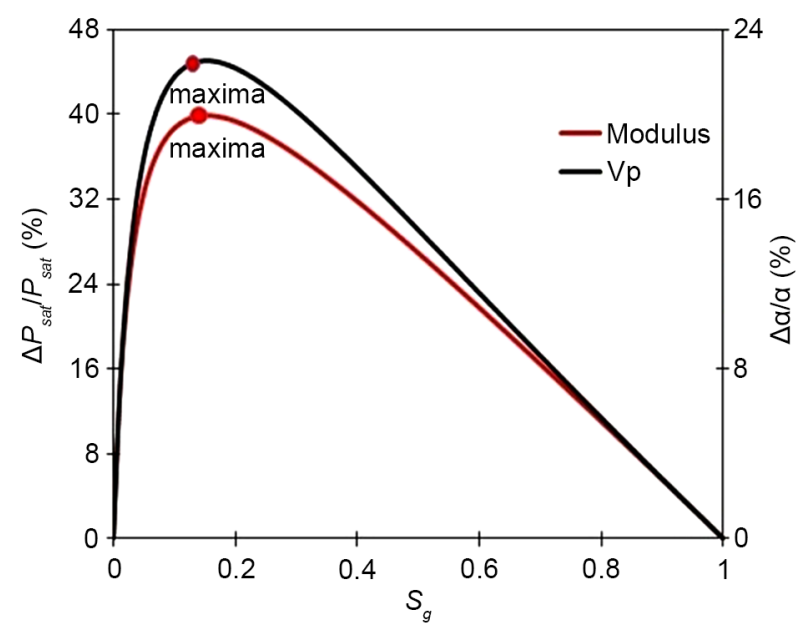

(b)

Figure 5. (a) $P$ wave modulus and velocity relative difference response for gas hydrate bearing sediments; (b) $P$ wave modulus and velocity relative difference response for gas bearing sediments.

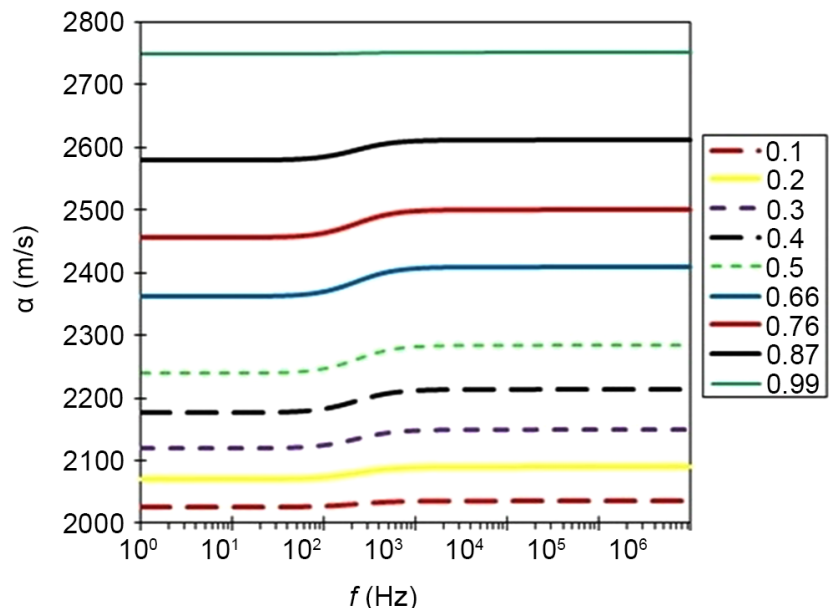

(a)

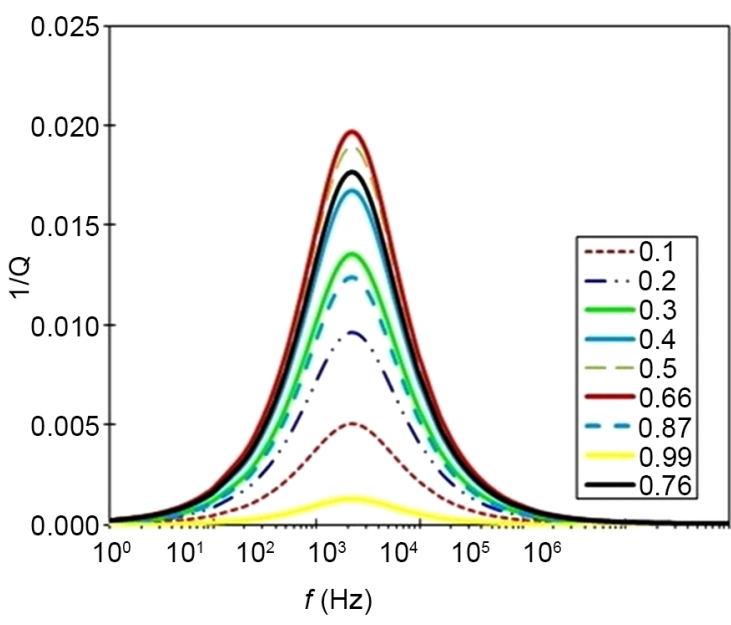

(b)

Figure 6. (a) Shows velocity dispersion response for gas hydrate bearing sediments at different saturation of gas hydrate bearing sediments; (b) It shows seismic attenuation for different gas hydrate saturated sediments.

gas hydrates. These patches assembled with each other in such a way that no fluid migrates from one patch to other patch. In (Figure 6(a)) we have plotted velocity dispersion for different gas hydrates saturation and maximum dispersion occurs at $66 \%$ gas hydrates. However, when sediments are fully saturated with gas hydrates (about $~ 0.99$ ) little attenuation can be observed. Seismic attenuation and velocity dispersion are directly interlinked with each other. In (Figure 6(b)), we have checked attenuation response for WIFF at seismic frequency to sonic frequency range. We have elaborated that maximum velocity dispersion and attenuation take place at $66 \%$ saturation of gas hydrates. We have seen from our results, with increase of gas hydrates saturation, velocity dispersion and attenuation also increased, when they are part of fluid. These phenomena of increasing attenuation with increase of gas hydrate saturation hold at certain limit of gas hydrates saturation; this certain limit is called characteristics saturation. After this characteristics saturation seismic attenuation decreases gradually.

The results reveal that when gas hydrates are part of fluid, seismic attenuation increase with increase in gas hydrates saturation until it will not reach at characteristics saturation limit. After this characteristics saturation the attenuation start to decrease.

We have also computed velocity dispersion and attenuation for gas saturated sediments as shown in (Figure 7). In (Figure 7(a)) velocity dispersion plots are computed for different gas saturation. Velocity dispersion plots 


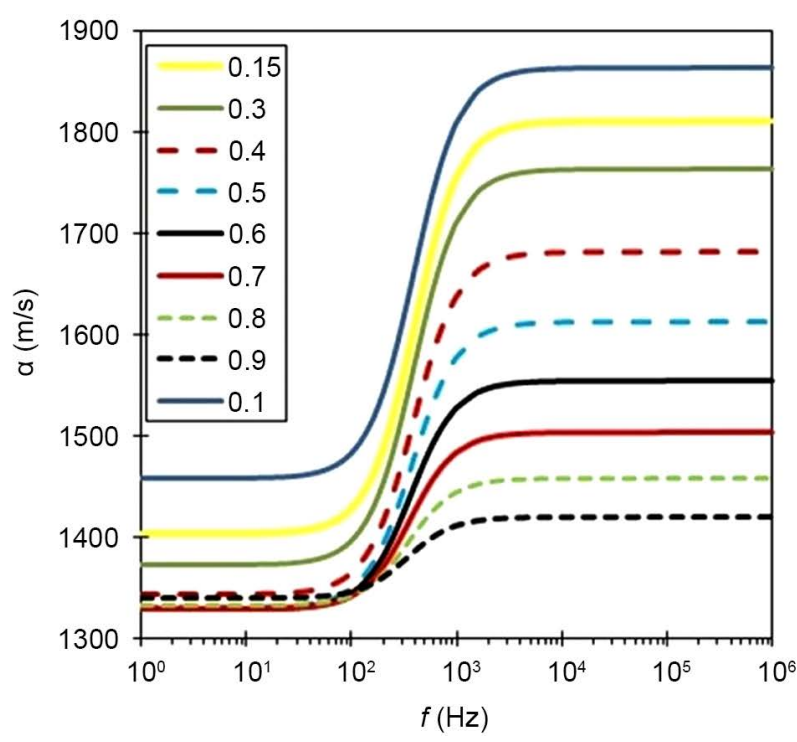

(a)

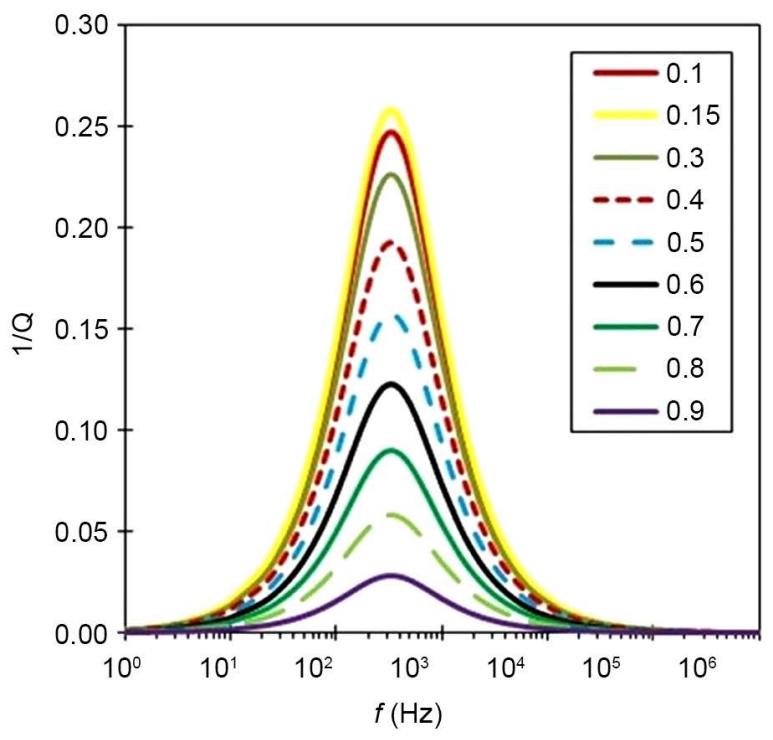

(b)

Figure 7. (a) Velocity dispersion plots for gas saturated zone in Makran offshore area Pakistan; (b) Seismic attenuation plots for gas saturated sediments.

show that maximum velocity dispersion for gas bearing sediments occurred at $15 \%$ gas saturation. Similarly maximum attenuation also obtained at $15 \%$ gas saturation as shown in (Figure 7(b)). Seismic attenuation and velocity dispersion is interrelated each other. In Figure 7(a) we have observed that maximum velocity dispersion is observed at $15 \%$ of gas saturation after this saturation velocity dispersion start decreasing. Similarly maximum attenuation is observed at $15 \%$ free gas saturation, after this saturation attenuation curves start decreasing with increasing gas saturation. Our all previous discussions elaborate attenuation and velocity dispersion for gas hydrate and gas bearing sediments shows that elastic properties of fluids and seismic response plays a vital role in attenuation and velocity dispersion phenomenon. We depicted that velocity difference $\Delta \alpha$ fluid difference $\Delta K_{f}$ and relative difference curves for $P$ wave velocity and $P$ wave effective modulus interconnected directly with attenuation. Seismic attenuation is an important attribute to depict low saturated gas reservoir and medial saturated gas hydrated reservoir for Makran offshore area Pakistan.

\section{Conclusion}

Seismic attenuation and velocity dispersion are interconnected with elastic properties of gas hydrates and gas bearing sediments. Velocity difference, fluid modulus difference and relative difference in $P$ wave modulus show maxima for gas hydrates and gas bearing sediments at $66 \%$ and $15 \%$ saturation. It is observed that when gas hydrates are part of fluid, seismic attenuation increases with increase of gas hydrate saturation, till characteristics saturation. After characteristics saturation, velocity dispersion and attenuation decrease for gas hydrate and gas bearing saturated zone. Seismic attenuation and velocity dispersion can be used as vital seismic attributes for the characterization of gas hydrates-bearing sediments and free gas saturated sediments for Makran offshore area Pakistan.

\section{References}

[1] Brooks, J.M., Kennicutt, M.C. and Fay, R.R. (1992) Thermogenic Gas Hydrates in Gulf of Mexico. Science, 225, 409-411. http://dx.doi.org/10.1126/science.225.4660.409

[2] Carroll, J.J. (2003) Natural Gas Hydrates, a Guide to Engineers. Gulf Professional Publishing, Amsterdam.

[3] Ginsburg, G.D., Guseynov, R.A. and Dadashev, A.A. (1992) Gas Hydrates of the Southern Caspian. International Geology Review, 42, 765-782. http://dx.doi.org/10.1080/00206819209465635

[4] Ecker, C., Dvorkin, J. and Nur, A. (1998) Sediments with Gas Hydrates: Internal Structure from Seismic AVO. Geophysics, 63, 1659-1669. http://dx.doi.org/10.1190/1.1444462 
[5] Hovland, M. and Judd, A.G. (1988) Sea Bed Pock-Marks and Seepages, Impact on Geology. Graham and Trotman Ltd., London, 422-438.

[6] Lowrie, A., Max, M.D., Hamiter, R., Lerche, I. and Bagirow, E. (1997) Hydrate Stability Zone Permanence along Dynamic Louisiana Offshore. Gulf Coast Association of Geological Societies Transactions, 47, 311-315.

[7] Marl, R.G., Bryan, G.M. and Ewing, J.L. (1970) Structure of Blake Bahamas outer Ridge. Journal Geophysical Research, 75, 4539-4555. http://dx.doi.org/10.1029/JC075i024p04539

[8] Stoll, R.D., Ewing, J.I. and Bryan, G.M. (1971) Anomalous Wave Velocities in Sediments Containing Gas Hydrates. Journal of Geophysical Research, 84, 15101-15116. http://dx.doi.org/10.1029/jb076i008p02090

[9] Chand, S. and Minshull, T.A. (2004) The Effect of Hydrate Content on Seismic Attenuation: A Case Study for Mallik 2L-38 Well Data, Mackenzie Delta, Canada. Geophysical Research Letter, 31, 2-5. http://dx.doi.org/10.1029/2004GL020292

[10] Gei, D. and Carcione, J.M. (2003) Acoustic Properties of Sediments Saturated with Gas Hydrate, Free Gas and Water. Geophysical Prospecting, 51, 141-157. http://dx.doi.org/10.1046/j.1365-2478.2003.00359.x

[11] Pecher, I.A. and Holbrook, W.S. (2003) Seismic Methods for Detecting and Quantifying Marine Methanehydrate/Free Gas Reservoirs. Natural Gas Hydrate, Springer, 275-294.

[12] Rossi, G., Gei, D., Böhm, G., Madrussani, G. and Carcione, J.M. (2007) Attenuation Tomography: An Application to Gas-Hydrate and Free-Gas Detection. Geophysics Prospect, 55, 655-669. http://dx.doi.org/10.1111/j.1365-2478.2007.00646.x

[13] Guerin, G. and Goldberg, D. (2002) Sonic Waveform Attenuation in Gas Hydrate-Bearing Sediments from the Mallik 2L-38 Research Well, Mackenzie Delta, Canada. Journal of Geophysical Research, 55, 107-110. http://dx.doi.org/10.1029/2001jb000556

[14] Bellefleur, G., Riedel, M., Brent, T., Wright, F. and Dallimore, S.R. (2007) Implication of Seismic Attenuation for Gas Hydrate Resource Characterization, Mallik, Mackenzie Delta, Canada. Journal of Geophysical Research, 112, B10311B10311. http://dx.doi.org/10.1029/2007jb004976

[15] Matsushima, J. (2006) Seismic Wave Attenuation in Methane Hydrate-Bearing Sediments: Vertical Seismic Profiling Data from the Nankai Trough Exploratory Well, Offshore Tokai, Central Japan. Journal of Geophysical Research, 111, B10101-B10101. http://dx.doi.org/10.1029/2005jb004031

[16] Sain, K., Singh, A.K., Thakur, N.K. and Khanna, R. (2009) Seismic Quality Factor Observations Forgas-HydrateBearing Sediments on the Western Margin of India. Marine Geophysical Research, 30, 137-145. http://dx.doi.org/10.1007/s11001-009-9073-1

[17] Li, C.H. and Liu, X. (2014) Analysis of P-Wave Attenuation in Hydrate-Bearing Sediments Based on Bisq Model. Geological Research.

[18] Ehsan, I.M., Ahmed, N., Khalid, P., Liu, X.W. and Naeem, M. (2015) An Application of Rock Physics Modeling to Quantify the Seismic Response of Gas Hydrate-Bearing Sediments in Makranaccretionary Prism, Offshore, Pakistan. Geosciences Journal, 20, 321-330. http://dx.doi.org/10.1007/s12303-015-0044-z

[19] Schluter, H.U., Prexl, A., Gaedicke, C., Roeser, H., Reichert, C., Meyer, H. and Daniels, C. (2002) The Makranaccretionary Wedge: Sediment Thicknesses and Ages and the Origin of Mud Volcanoes. Marine Geology, 185, 219-232. http://dx.doi.org/10.1016/S0025-3227(02)00192-5

[20] Khalid, P. and Ahmed, N. (2016) Modulus Defect, Velocity Dispersion and Attenuation in Partially-Saturated Reservoirs of Jurassic Sandstone, Indus Basin, Pakistan. Studia Geophysica Geodetica, 60, 112-129. http://dx.doi.org/10.1007/s11200-015-0804-2

[21] Helgerud, M.B., Dvorkin, J. and Nur, A. (1999) Elastic Wave Velocity in Marine Sediments with Gas Hydrates. Geophysical Research Letter, 26, 2021-2024. http://dx.doi.org/10.1029/1999GL900421

[22] Khalid, P., Broseta, D. and Nichita, D.V. (2014). A Modified Rock Physics Model for Analysis of Seismic Signatures of Low Gas-Saturated Rocks. Arabian Journal of Geosciences, 7, 3281-3295. http://dx.doi.org/10.1007/s12517-013-1024-0

[23] Wood, A.B. (1941) A Textbook of Sound. G. Bell and Sons.

[24] Reuss, A. (1929) Berechnung der fliessgrense von mischkristallen auf grund der plastizitatbedingung fur einkristalle. Zeitschrift Fur Angewandte Mathematik Und Mechnik, 25, 49-58. http://dx.doi.org/10.1002/zamm.19290090104

[25] Gassmann, F. (1951) Uber die Elastizit a tporoserMedien. Vierteljahrsschriftder aturforschendenGesellschaft in Zurich, 96, 1-23.

[26] Khalid, P. (2011) Effects on Seismic Properties of Thermo Elastic Relaxation and Liquid/Vapor Phase Transition. PhD Dissertation, Pau University France, Pau. 
[27] Hill, R. (1963) Elastic Properties of Reinforced Solids: Some Theoretical Principles. Journal of the Mechanics and Physics of Solids, 11, 357-372. http://dx.doi.org/10.1016/0022-5096(63)90036-X

[28] Gosh, R. and Sain, K. (2008) Effective Medium Modeling to Assess Gas Hydrate and Free-Gas Evident from the Velocity Structure in the Makran Accretionary Prism, Offshore Pakistan. Marine Geophysics, 29, 267-274.

http://dx.doi.org/10.1007/s11001-009-9062-4

\section{Submit or recommend next manuscript to SCIRP and we will provide best service for you:}

Accepting pre-submission inquiries through Email, Facebook, LinkedIn, Twitter, etc.

A wide selection of journals (inclusive of 9 subjects, more than 200 journals)

Providing 24-hour high-quality service

User-friendly online submission system

Fair and swift peer-review system

Efficient typesetting and proofreading procedure

Display of the result of downloads and visits, as well as the number of cited articles

Maximum dissemination of your research work

Submit your manuscript at: http://papersubmission.scirp.org/ 